\title{
Deformities of the hands and feet in Parkinsonism and their reversibility by operation
}

\author{
PETER GORTVAI \\ From the National Hospital for Nervous Diseases, Queen Square, London
}

Charcot (1877) described some aspects of the typical deformities of the hands and feet seen in Parkinson's disease. He likened the deformity of the hands to that sometimes observed in chronic progressive rheumatism. He said that the differential diagnosis is usually made with ease, because in cases of Parkinsonism 'there is found neither the articular tumefaction and stiffness, nor the osseous deposits and cracking sounds observed in nodose rheumatism'. $\mathrm{He}$ describes the deformity of the toes as a 'griffe' (or claw), on account of the extension of the first and concomitant flexion of the second phalanges. Hughlings Jackson (1899) also mentions deformity of hands seen in some cases of Parkinsonism. He remarked that 'in paralysis agitans the interossei are the muscles of the hand which are preponderatingly affected and the set of the hand resembles that of tetany of children'. Cooper and Bravo (1958) show that the deformity of the hands in a case of Parkinsonism was relieved by unilateral occlusion of the anterior choroidal artery.

The deformities of the hands and feet add to the difficulties of severely handicapped patients. In suitable cases most of these deformities are reversible by operation on the thalamus.

The typical deformities will be described, their mode of production discussed, and it will be shown that they are reversible by stereotactic lesions of the thalamus.

\section{CLINICAL MATERIAL}

One hundred and fifty cases of Parkinsonism of all types have been treated by operation under the care of Mr. Harvey Jackson. The deformities of the hands and feet were not uncommon in the fairly severely affected patients. They were more commonly seen in those showing rigidity in excess of tremor and they often exhibited bradykinesia and paucity of voluntary movement. Operations on the ventrolateral nucleus of the thalamus were performed by the method described by McCaul (1959), slightly modified by us in some details. This is a stereotactic method using a small instrument which screws directly into the skull and depends on localization of the target in the brain on the measurement of angles in two planes. The lesions in the ventrolateral nucleus of the thalamus were made by the injection of 0.5 to $1 \mathrm{ml}$. of a mixture of Etopalin (Ciba) and kaolin.

\section{DESCRIPTION OF THE DEFORMITIES}

THE HANDS In its early and commonest stage the deformity amounts to no more than a characteristic posture of the affected hand (Fig. 1). The fingers are held extended at the interphalangeal joints and flexed some $40^{\circ}$ to $70^{\circ}$ at the metacarpo-phalangeal joints. The thumb is extended at both joints and it is adducted and opposed. The hand is often held in slight ulnar deviation.

The next stage with more advanced disease is characterized by hyperextension of the fingers at the interphalangeal joints (Fig. 2).

In the third stage of the deformity, not often seen, the hyperextension is so severe and chronic that subluxation occurs at the distal interphalangeal joints. With the subluxation the terminal phalanges are first in extension but with the passage of time they are in flexion, while the proximal interphalangeal joints become even more hyperextended (Fig. 3).

Through these stages of deformity of the hands there is increasing weakness of voluntary movement and bradykinesia.

THE FEET Clawing of the toes is not uncommon. Sometimes all toes, apart from the big toe, are in flexion, crowded together, producing a painful cramp. As the toes curl under the ball of the foot they impede the gait of the patient who already has considerable difficulty in walking due to other features of Parkinsonism. The foot is generally held in inversion and taking festinating steps on the outer border of the foot can be really troublesome, especially as so many of the patients have difficulty in maintaining balance. 


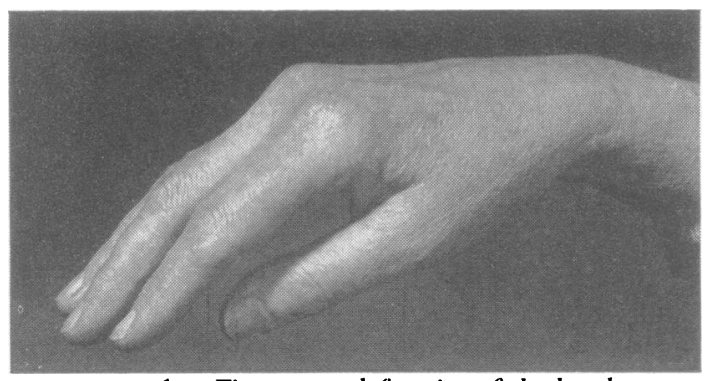

FIG. 1. First stage deformity of the hand.

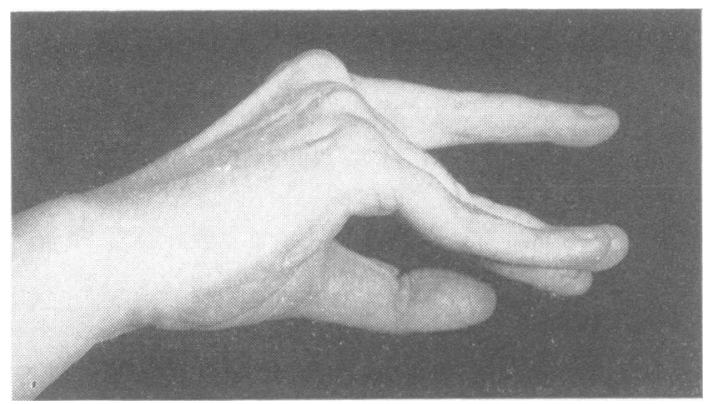

FIG. 2. Second stage deformity of the hand.

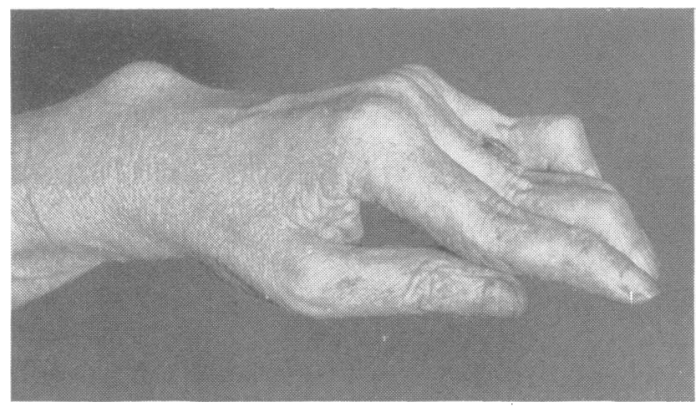

FIG. 3. Third stage deformity of the hand.

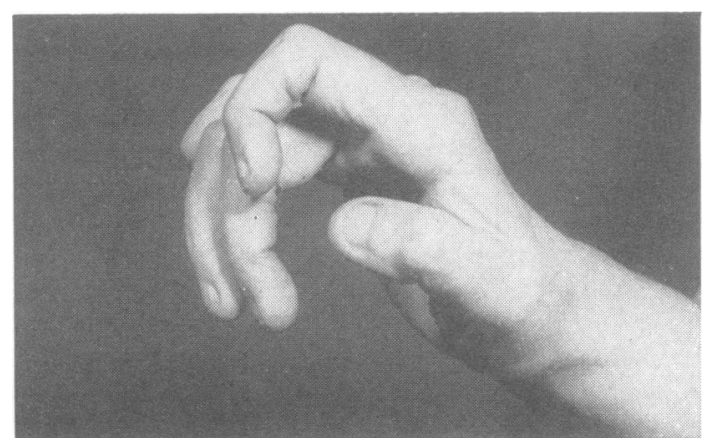

FIG. 4. Normally flexed fingers of the hand shown in Fig. 2 after temporary ulnar palsy.
MODE OF PRODUCTION OF THE DEFORMITIES

As Charcot says, the deformities of the hand can be differentiated from chronic arthritis by the absence of other local signs of joint involvement. The joints indeed are healthy both on clinical and radiological examination. It is remarkable how healthy these joints can be radiologically after many years of deformed posture.

The ligaments of the small joints remain healthy until the last stage of the deformity of the hands when subluxation of the terminal phalanges occurs. The integrity of the ligaments is shown by the fact that in spite of the deformity there is never any unnatural mobility in any of the joints apart from the terminal interphalangeal ones.

The deformities are caused by muscular rigidity which is one of the characteristic features of Parkinsonism. The rigidity is maintained by a reflex arc, as was shown by Walshe (1924).

The role of contraction of the small muscles in the hand in the production of deformities can be shown by the abolition of the deformities on blocking the ulnar nerve with local anaesthetic and by reproducing the characteristic posture of the hand on stimulation of the ulnar nerve in an unaffected person.

Infiltration of $5 \mathrm{ml}$. of $1 \%$ procaine around the ulnar nerve as it is lying on the flexor retinaculune causes paralysis of most of the small muscles of the hand. When the effect is of sufficient intensity the deformed fingers assume the normal anatomica position of function, and movement of the digits by the intact long flexors and extensors becomes easier and faster. Figure 4 illustrates the correction of the extension deformity shown in Fig. 2 during temporary ulnar paralysis.

During operations for transposition of the ulnar nerve for lesions in the region of the elbow joint the opportunity was taken to study the effect of stimulation of the ulnar nerve on the posture of the fingers. After exposure of the nerve in the region of the elbow joint the course of the nerve was demonstrated in the upper part of the forearm by splitting the flexor carpi ulnaris muscle into fibres deriving from its two heads of origin. A dipolar electrode was applied to the nerve distal to the branches innervating the flexor carpi ulnaris and the flexor digitorum profundus. The only motor fibres stimulated in this way were those to the small muscles of the hand. Square impulses were applied to the nerve with the frequency of 50 per second at 6 volts.

When the nerve was stimulated in a patient with only sensory signs and symptoms and no muscular weakness, the characteristic flexion at the metacarpophalangeal joints and extension at the interphalangeal joints was reproduced. Extension occurred at 
all the interphalangeal joints. This was probably caused by contraction of the interossei because only those small hand muscles are attached to all the fingers. Flexion at the metacarpo-phalangeal joint was observed in the medial two fingers, showing that the flexion is due to contraction of the lumbricals. In the true Parkinsonian posture all fingers are flexed at the metacarpo-phalangeal joint, but all muscles are in a state of increased tone, not only those supplied by the ulnar nerve. The characteristic posture of the thumb, extension at both its joints with slight adduction and opposition, is probably produced by the increased tone of the small muscles contained in the thenar eminence. Many of these are supplied by the median nerve and they were not affected by the experiments on the ulnar nerve.

\section{REVERSIBILITY OF THE DEFORMITIES} BY STEREOTACTIC SURGERY

Surgical lesions suitably placed in the ventrolateral nucleus of the thalamus can alleviate the tremor and rigidity of Parkinsonism. The largest series was described by Cooper (1961). With the improvement of rigidity the long-standing deformities of the hands and feet are also corrected and there is immediate improvement in muscle power and in the speed of voluntary movement. Figure 5 shows the improved posture of the right hand after operation, the same hand as was shown pre-operatively in Figure 2.

Our cases have now been followed up for one to three years post-operatively. Relief from the deformities seems to be permanent so far.

In the selection of patients for operation difficulty in walking is often regarded as a point against the decision to operate because the sense of balance is sometimes disturbed by the stereotactic procedure and standing and walking made worse. The difficulty in gait should be carefully observed because it may

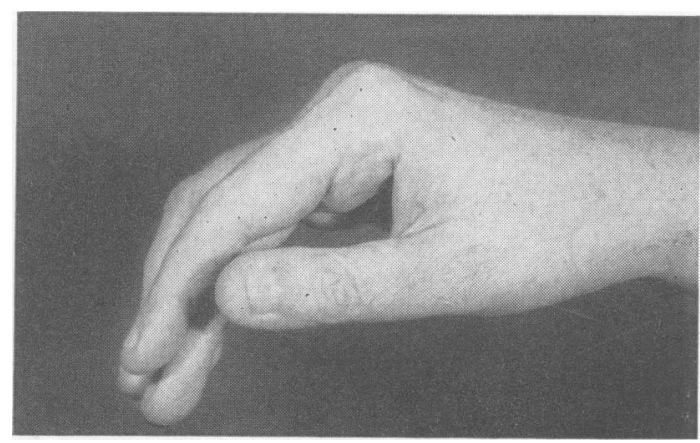

FIG. 5. Corrected posture of the hand shown in Fig. 2 after thalamotomy.

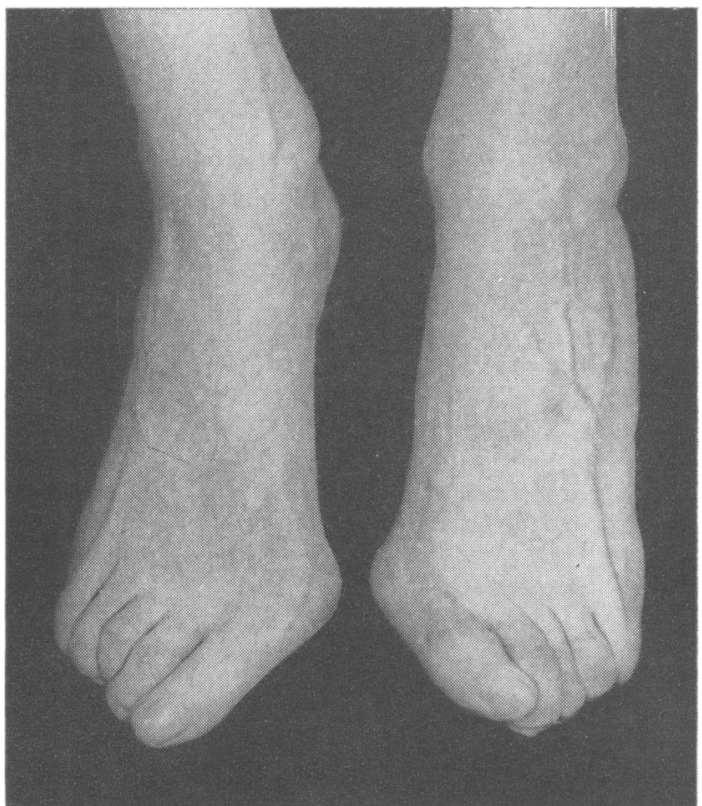

FIG. 6. Deformed feet before operation, with the toes curled under, nails facing the ground, and painful callosities over the distal joints.

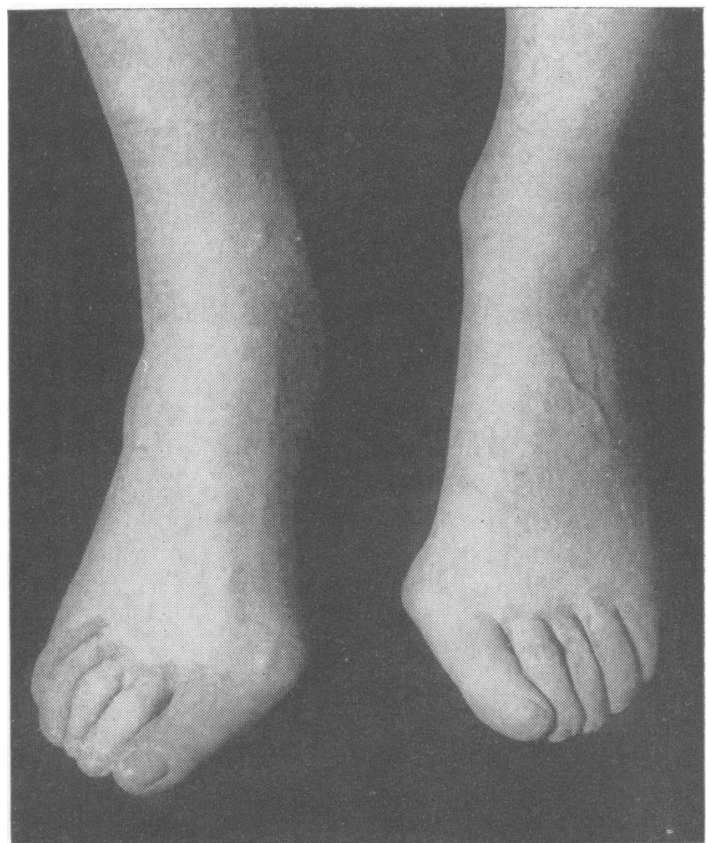

FIG. 7. The same patient's feet as in Fig. 6 after bilateral thalamotomy. The toes are much straighter, apart from bilateral hallux valgus. 
be caused by akynesia, rigidity, or by deformities of the feet and not by a disturbed sense of balance. In such cases the operation may improve the patient's gait.

The relief experienced by a patient afflicted by deformities and cramps of the feet is illustrated by the following case report:-

A 54-year-old housewife (hospital No. 90683) was admitted to the National Hospital for Nervous Diseases in April 1960. She had had a 'flu-like' illness accompanied by severe headache in 1942. Since 1945 she had been suffering from typical Parkinsonian tremor of the right upper limb. A year later she began to have difficulty in walking as she tended to stumble over the right foot. She developed painful cramps in the right foot. At the time of her admission she could just about manage to walk on even ground unaided but stumbled as soon as there was the slightest irregularity. On uneven ground she could not even stand without assistance.

On examination she had a rather immobile face and her voice was monotonous. There was fairly severe Parkinsonian tremor and rigidity in the right limbs. Muscle tone was also increased in the left limbs. Her toes were bunched up and curled under (Fig. 6), more severely on the right side.

A stereotactic lesion was made in the ventrolateral nucleus of the left thalamus in April 1960. The tremor and rigidity of the right limbs were satisfactorily alleviated and the patient was able to walk much better than before operation. She volunteered the information that the painful cramps in the right foot had disappeared.

Six months after operation the rigidity of the left limbs started to become increasingly more severe and she developed a tremor of the left hand. She also began to complain of painful cramps, this time in the left foot. The ventrolateral nucleus of the opposite cerebral hemisphere was operated on in April 1961 and great improvement followed in the tremor and rigidity. The cramps in the foot had disappeared and she was walking well. The toes were much straighter than before the operations and the painful callosities (Fig. 7) over the terminal joints had disappeared.

The patient was last seen in April 1962. She had only a slight residual tremor of the right hand but apart from that there was no tremor and practically no rigidity. Her feet felt quite comfortable and she walked quite normally.

\section{SUMMARY}

Characteristic deformities of the hands and feet are described in cases of Parkinson's syndrome.

The deformities are due to muscular rigidity. This was shown by the abolition of the deformities on the induction of temporary ulnar paralysis and the reproducibility of the characteristic posture by stimulation of the ulnar nerve.

There is no clinical or radiological evidence of joint disease even in advanced cases.

The deformities can be associated with painful muscle cramps. Deformities of the feet may impede gait.

The deformities are lastingly reversible by operation on the ventrolateral nucleus of the thalamus.

A case of post-encephalitic Parkinsonism is described. Bilateral thalamotomy relieved painful cramps in the feet, straightened the toes, and improved gait.

I wish to thank Mr. Harvey Jackson for his interest advice, and permission to operate on a number of his patients, Dr. John Bates for his help with electricad stimulation, and the Medical Committee of the Nationaf T Hospital, Queen Square, for permission to publish this paper.

\section{REFERENCES}

Charcot, J. M. (1877). Lectures on the Diseases of the Nervous System, translated by George Sigerson. The New Sydenham Society, London.

Cooper, I. S. (1961). Parkinsonism-its Medical and Surgical Therapy. Thomas, Springfield, Illinois.

- and Bravo, G. J. (1958). In Pathogenesis and Treatment of Parkinsonism: Sixth Annual Scientific Meeting of the Houston Neurological Society, Texas Medical Center, Houston, Texas, ed. W. S. Fields, p. 325. Thomas, Springfield, Illinois.

Jackson, J. Hughlings (1899). Brain, 22, 621.

McCaul, I. R. (1959). J. Neurol. Neurosurg. Psychiat., 22, 109.

Walshe, F. M. R. (1924). Brain, 47, 159. 